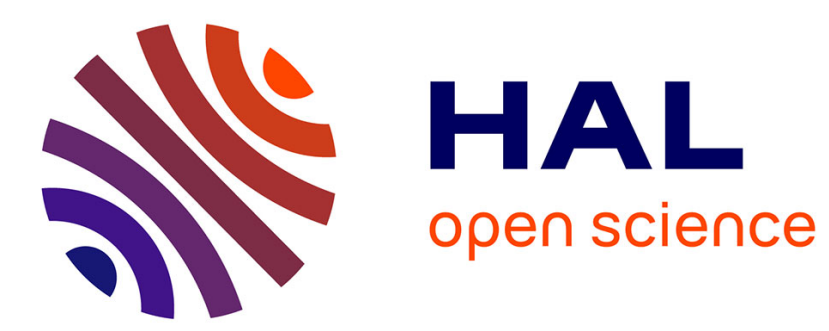

\title{
PROGRÈS RÉCENTS DANS LA FABRICATION DES LAITS ENTIER ET CONCENTRÉS STÉRILES
}

\author{
Ernest O. Herredd
}

\section{To cite this version:}

Ernest O. Herredd. PROGRÈS RÉCENTS DANS LA FABRICATION DES LAITS ENTIER ET CONCENTRÉS STÉRILES. Le Lait, 1956, 36 (351_352), pp.14-26. hal-00928154

\section{HAL Id: hal-00928154 \\ https://hal.science/hal-00928154}

Submitted on 1 Jan 1956

HAL is a multi-disciplinary open access archive for the deposit and dissemination of scientific research documents, whether they are published or not. The documents may come from teaching and research institutions in France or abroad, or from public or private research centers.
L'archive ouverte pluridisciplinaire HAL, est destinée au dépôt et à la diffusion de documents scientifiques de niveau recherche, publiés ou non, émanant des établissements d'enseignement et de recherche français ou étrangers, des laboratoires publics ou privés. 
[20] R. Richou et G. Holstein. Bull. Acad. Vét., 1938, 11, 158.

[21] G. Holstein et R. Richоu. Bull. Acad. Vét., 1940, 13, 193. - Rec. Méd. Vét., 1941, 117, 116.

[22] R. Richou et G. Holstein. Rec. Méd. Vét., 1941, 117, 11.

[23] R. Richou, G. Holstein et J. Renauldon. C. R. Soc. Biol., 1941, 135, 789 .

R. Richou et G. Holstein. Rev. Imm., 1941, 6, 363.

[24] Murphy. The Vet. Record, 1946, 58, 277.

[25] G. Ramon, R. Richou, P. Julienne, J. Jacquet et Cl. Gerbeaux. Bull. Off. Int. Epiz., 1952, 37, 145. - C. R. Acad. Sc., 1952, 234, 28. - Rev. Imm., 1951, 15, 321.

[26] R. Riohou, J. Jacquet, P. Julienne et Cl. Gerbeaux. Rev. Path. Comp. et Hyg. gén., 1952, 52, 231. - C. R. Soc. Biol., 1952, 146, 636.

[27] G. Ramon, R. Richou, J.-P. Thíkry, Cl. Gerbeaux et J. Leplatre. C. R. Acad. Sc., 1949, 229, 278. - Rev. Imm., 1949, 13, 301. - C. R. Acad. Sc., 1950, 231, 563. - Rev. Imm., 1950, 14, 205.

[28] R. Richou, Cl. Gerbeaux et Mile J. Schlaepfer. C. R. Acad. Sc., 1949, 229, 858 .

[29] G. Ramon, R. Richou, P. Julienne, J. Jacquet, Cl. Gerbeaux et G. Leneveu. Bull. Off. Int. Epiz., 1952, 37, 514.

[30] G. Ramon, R. Richou, P. Julienne et J. Jacquet. Bull. Off. Int. Epiz., $1953,39,610$.

G. Ramon, R. Richou, J.P. Thiéry et Cl. Gerbeaux. C. R. Aead. Sc., 1952, 234, 2132. - Bull. Off. Int. Epiz., 1952, 37, 228.

[31] G. Ramon, R. Richou, G. Delaunay et Cl. Gerbeaux. C. R. Acad. Sc., 1952, 324, 1657.

[32] G. Ramon, R. Richou et Cl. Gerbeaux (l'association de la pénicillinothérapie à l'anatoxithérapie staphylococcique, préconisée par G. Ramon et R. RICHov, dès 1945 , ne gêne en rien l'apparition et le développement de l'immunité antistaphylococciq̊ue). C. R. Acad. Sc., 1951, 233, 121.

\title{
PROGRÈS RÉCENTS DANS LA FABRICATION DES LAITS ENTIER ET CONCENTRÉS STÉRILES (1)
}

\author{
par Ernest O. HERREID
}

Station Expérimentale Agricole de l'Illinois, Urbana (Illinois).

Le lait stérile ou pratiquement stérile est en évidence en Angleterre et en Europe depuis plus de cinquante ans. Dans certaines régions d'Angleterre, une troisième génération en boit, alors que certains pays étrangers en achètent pour les enfants et les invalides. Il fut pendant de nombreuses années le seul lait disponible qui ne soit pas dangereux. Il répondait à pẹu près aux mêmes besoins que

(1) Conférence donnée lors de la $47^{\circ}$ Convention annuelle de Milk Industry Foundation, tenue à Atlantic City, N. J., (Le Québec laitier, 1955, nº 8, 12 ). 
le lait certifié de chez nous, avant la pratique généralisée de la pasteurisation. Le lait stérile convient aux pays où la plupart des maisons n'ont pas de réfrigérateur.

Le lait frais, concentré et pasteurisé, apparut sur le marché en 1933, dans plusieurs villes de notre pays. En 1950 et 1951, il revint à nouveau dans de nombreuses cités, appuyé par tous les moyens de publicité. Mais la population ne l'accepta pas d'une façon permanente, surtout parce qu'il se vendait au même prix que le lait de consommation régulier. Néanmoins, le lait concentré frais fait sa réapparition dans certaines régions. Comme le lait entier, on peut le stériliser rapidement, l'embouteiller ou l'emboîter aseptiquement; reconstitué, il fournit un lait acceptable, grandement supérieur au lait évaporé stérilisé en boîte.

\section{Orientation de l'industrie laitière}

L'industrie laitière, en ce qui concerne le traitement des produits liquides, tend vers les opérations continues, l'utilisation de températures plus élevées et de durées d'exposition plus courtes. Elle a comme objectifs : l'amélioration de la qualité de conservation, le maintien et la préservation des propriétés nutritives et de la sapidité des produits, et l'économie de temps et de travail. Les températures de pasteurisation industrielle du lait montent continuellement.

Quand les standards de pasteurisation du lait de consommation furent formulés, on tint compte de quatre facteurs :

$1^{\circ}$ La destruction des bactéries pathogènes;

$2^{\circ}$ La plus grande conservation possible de la saveur naturelle du lait ;

$3^{\circ}$ La conservation de la ligne ou du volume de crème; et

$4^{\circ}$ La conservation prolongée de la saveur agréable du lait.

En ces dernières années, la ligne naturelle de crème et la saveur naturelle du lait eru ont perdu de leur importance. Pratiquement, tout le lait est maintenant homogénéisé et, dans plusieurs localités, les consommateurs se sont graduellement habitués à la saveur de cuit ou de chauffé dans le lait. En certains endroits où l'on utilise des appareils à haute température, le lait est chauffé jusqu'à $178^{\circ} \mathrm{F}$. (1) pendant 15 secondes, au lieu de la température officielle de $161^{\circ} \mathrm{F}$. Cette pratique a tellement amélioré la conservation de la saveur du lait de consommation que ce dernier a pu subir sans

(1) Pour convertir en degrés centigrades, il convient de retrancher 32 , puis de multiplier par $\frac{5}{9}$. Exemple : $178^{\circ} \mathrm{F}$. $=(178-32) \frac{5}{9}=81^{\circ} 1 \mathrm{C}$. 
dommage la distribution et l'entreposage à des distances considérables de l'usine. Une récente enquête portant sur des laits de consommation provenant de 22 laiteries équipées d'appareils à haute température révéla qu'ils possédaient tous des saveurs de euit ou de chauffé à divers degrés. Cela n'est pas grave et, au surplus, est inévitable quand on fait usage de si hautes températures.

Plusieurs consommateurs de lait et de beurre ont accepté cette saveur. Des juges de lait expérimentés ont marqué leur préférence pour le lait traité à haute température, parce qu'elle conférait au produit ce qu'ils appellent une saveur de cuit amandé. Cette dernière tend à disparaître pendant l'entreposage. L'histoire prouve que les gens s'habituent à la saveur de cuit, ainsi qu'il est évident en Angleterre où se consomme du lait stérile ou presque depuis environ soixante ans et où les méthodes de chauffage impriment un goût prononcé de cuit ou de caramel. Cependant, un changement brusque de la saveur du lait pasteurisé régulier à la saveur prononcée de cuit est de nature à semer de la suspicion parmi les consommateurs concernant la qualité des laits d'approvisionnement. Les gens ne s'habituent que graduellement aux changements de saveur, comme ce fut le cas en maints endroits ici et outre-mer.

Dans notre pays, l'industrie beurrière a fait œuvre de pionnier dans la pasteurisation de la crème à des températures plus élevées que celles spécifiées par le Service de Santé publique des Etats-Unis. Le Canada, la Nouvelle-Zélande, l'Australie et le Danemark ont également utilisé les hautes températures de pasteurisation et cette pratique s'est révélée juste et bonne, puisque le beurre possédait des qualités supérieures de conservation tant au point de vue bactériologique qu'au point de vue chimique. Les usines de crème glacée emploient maintenant des températures de pasteurisation plus hautes avec des durées de retenue plus courtes, tout en conservant au produit un corps et une texture désirables. Nous nous attendons même à ce qu'elles en utilisent encore de plus hautes quand les autorités de santé permettront l'usage d'outillage moderne à haute température.

Le lait entier est l'un des derniers de nos principaux aliments périssables à connaître la stérilisation. Trois raisons expliquent la chose :

10 Le lait n'est pas un produit saisonnier comme les fruits et les légumes. Sa production est quotidienne, quoique des surplus apparaissent au début de l'été ;

$2^{\circ}$ L'industrie du lait de consommation est organisée pour traiter et distribuer un lait pasteurisé sain aux consommateurs et aux détaillants; 
$3^{\circ}$ L'équipement pour stériliser le lait n'est disponible que depuis peu de temps.

\section{Méthodes de stérélisitation du lait}

Ce sujet des méthodes de stérilisation est trop vaste pour le traiter adéquatement dans cette étude. Nous nous bornerons à décrire brièvement les méthodes générales employées pour le lait et quelques-uns de leurs dérivés.

\section{Méthode par injection de vapeur.}

Le lait est préchauffé à $120-140^{\circ} \mathrm{F}$. et reçoit ensuite une injection directe de vapeur, sa température s'élevant à 280-300 $\mathrm{F}$. en 0,5 à 1 seconde. Le lait se dirige ensuite vers une chambre à vide où la vapeur condensée et les mauvaises saveurs sont enlevées. On prétend qu'une certaine homogénéisation se produit dans le chambre à vide. Récemment, un appareil de ce genre a été mis au point en Suisse et où ce procédé de stérilisation a pris le nom de " upérisation ". En plus de la vapeur d'eau, on soutient que le gaz carbonique chauffé ou l'azote pourraient également servir dans cet appareil à la stérilisation de certains aliments liquides. D'autres chercheurs ont créé un instrument analogue. Le chauffage par injection de vapeur n'est pas nouveau. Des brevets ont été émis en faveur du procédé Grindrod il y a environ vingt ans, et un certain nombre d'autres brevets ont paru au cours des dernières années.

\section{Stéritisation én bouteilles.}

En Angleterre et en Europe, le lait est stérilisé ou pratiquement stérilisé en bouteilles de verre. On le préchauffe à $160-170^{\circ} \mathrm{F}$., l'homogénéise à 2,500 livres au po. c. et l'embouteille. Les bouteilles vides avaient préalablement subi le lavage et étaient à peu près à la même température que le lait. Les contenants, une fois remplis, sont scellés à l'aide d'une capsule-couronne, chauffés à $212^{\circ} \mathrm{F}$., puis dirigés dans une chambre à vapeur où ils demeurent pendant 30 minutes à $224-226^{\circ}$. Les bouteilles refroidissent en passant dans un bain à $212^{\circ} \mathrm{F}$. et, par la suite, baissent graduellement de température dans de I'eau à $70^{\circ} \mathrm{F}$. Le lait stérile possède une saveur de cuit, de caramel, Les bouteilles de lait ne sont pas toutes stériles, mais leur qualité de conservation est suffisante pour supporter les transports non réfrigérés et se garder à la maison pendant une semaine ou dix jours.

Stérilisation en boite.

La Suède a développé un procédé assez simple par lequel les boîtes remplies de lait passent dans un tunnel aérodynamique 
chauffé à l'électricité qui coûte peu dans ce pays. Le lait dans les boîtes atteint les environs de $300^{\circ} \mathrm{F}$. et se refroidit dans un tunnel du même genre mais réfrigéré ou encore dans un bain d'eau. La saveur de ce lait semble donner satisfaction aux consommateurs suédois. Une méthode semblable est à l'essai aux Etats-Unis. Le procédé Winger est une méthode modifiée de stérilisation en boîte, avec cette particularité qu'avant la stérilisation le lait est ehauffé avec du peroxyde d'hydrogène dont on enlève l'excès par l'addition de catalase. Dans le stérilisateur, les boîtes tournent continuellement sur elles-mêmes, ce qui accélère le chauffage en forçant les bulles de vapeur et d'air à traverser la masse du lait pour atteindre l'espace vide du dessus.

\section{Les échangeurs thermiques.}

Hanrahan créa un chauffeur en spirale cannelée pour lequel il reçut, en 1948, un brevet. La vitesse d'écoulement peut varier de 13 à 25 pieds par seconde. Cet appareil a déjà servi commercialement. La Chicago Stainless Steel Corp. a inventé récemment un appareil du même genre, appelé " pasteurisateur H.T.S.T. Roswell ». Il a été utilisé expérimentalement pour le mélange à crème glacée à la Station Expérimentale Agricole de l'Illinois. Une compagnie en fait l'essai avec du lait. Un chauffeur à lames rotatives qui hâtent l'échange thermique et préviennent le surchauffage fut mis au point par la Girdler Corp, et essayé avec du lait et de la crème.

La Station Expérimentale Agricole de l'Illinois se sert du chauffeur tubulaire à petit orifice Mallory. Cette unité comprend trois sections de chauffage indépendantes et trois sections de refroidissement. Les tubes d'acier inoxydable de la section chauffante comme celle de refroidissement ont un diamètre d'un quart de pouce et 142,7 pieds de longueur.

Par les méthodes d'échange thermique et d'injection de vapeur, le lait peut être rapidement chauffé aux températures de stérilisation avec peu d'effets sur sa saveur et ses autres propriétés. Mais il faut l'emboîter aseptiquement. Le procédé de mise en boîte aseptique Martin convient aux produits laitiers liquides, aux soupes et aux jus de fruit. Il utilise les boittes métalliques à service unique. Dans une usine d'essai de l'ouest, on expérimente la possibilité pratique de remplir aseptiquement les bouteilles de verre. De bonnes raisons nous permettent de croire la chose possible.

Il faut prévoir beaucoup de développement dans le domaine de l'outillage servant à la stérilisation et à l'embouteillage aseptique du lait et de ses dérivés. Ce sont là de nouveaux horizons dans le monde des industries laitière et alimentaire. 


\section{Expériences poursuivies à la Station Expérimentale Agricole de l'Université de l'lllinois}

Nous travaillons depuis quatre ans sur la préparation des produits laitiers stériles dans une usine d'essai. Le plus gros des efforts ont porté sur le lait entier et le lait concentré, mais quelques travaux ont aussi été faits avec de la crème. Le premier problème rencontré fut celui de l'élimination, aussi complète que possible, de l'effet des températures de stérilisation sur la saveur du lait. La chose s'est révélée moins difficile que prévue. En ajustant la pression de la vapeur dans la section chauffante du chauffeur Mallory, il a été possible de stériliser le lait en ne lui donnant qu'un léger goût de cuit qui disparaissait après quelques jours. Certains des juges n'ont mềme pu déceler aucune saveur de cuit dans un lait fraîchement stérilisé.

Le problème suivant consistait à recueillir aseptiquement le lait sortant du chauffeur Mallory. On y arriva en soudant quatre pouces de tuyau de $1 / 2$ " de diamètre muni d'un manchon, au eouvercle d'un bidon de dix gallons, et en raccordant le manchon à un coude du circuit de la section de refroidissement pour recevoir le lait stérile. Le bidon possédait une ouverture prolongée d'un tube de caoutchoue conduisant le liquide dans les bouteilles. Tout cet assemblage était stérilisé dans un autoclave. On stérilisait le Mallory avec de l'eau à $300^{\circ} \mathrm{F}$. environ. Des éléments thermo-électriques placés à divers points du circuit à intervalles d'environ 0,5 de seconde assuraient la température finale à laquelle le lait était chauffé. L'appareil est entièrement fait d'acier inoxydable.

Le problème final avait trait à l'embouteillage aseptique du lait. On fabriqua une chambre en contreplaqué qui mesurait 5 pieds de long (le devant), 4 pieds de haut et 2 pieds de large, avec une fenêtre à l'avant qui permettait à deux techniciens de voir le travail qu'ils exécutaient. La chambre contenait une capsuleuse à main. Du bidon, le lait coulait dans environ trois pieds de tube caoutchouté stérile et pénétrait dans la chambre aseptique. Une personne emplissait la bouteille de verre stérile, l'autre saisissait la capsule avec un aimant, la plaçait sur le contenant et scellait avec une capsuleuse à main. Les deux personnes travaillaient au travers de quatre ouvertures circulaires pratiquées dans le mur avant, mains et avant-bras recouverts d'un long gant caoutchouté de chirurgien. La partie ouverte de chaque gant était attachée au mur de la chambre et tenue en place par une rondelle en bois fixée avec un écrou à oreilles. Avant l'embouteillage, tout l'équipement, y compris les bouteilles et les capsules stériles, était placé dans la chambre et protégé contre le chlore. On vaporisait ensuite la 
chambre avec 15 à 20 millilitres d'eau chlorée contenant environ $1,000 \%$ de chlore.

L'efficacité aseptique de cette méthode fut mesurée en premier en embouteillant du bouillon nutritif préalablement stérilisé dans le bidon de 10 gallons. Chaque bouteille de bouillon a manifesté sa stérilité après une longue incubation à 40,70 et $100^{\circ} \mathrm{F}$. Les pertes de lait s'élevèrent à moins de $0,2 \%$ dont une partie pourrait être attribuée à l'inexpérience des techniciens. Récemment, on remplaça la chambre de bois par une autre en "lucite", ce qui comportera des améliorations que nous expliquerons dans une prochaine publication.

Dans la plupart des expériences, nous avons employé le lait de la ferme de l'Université. Il était chauffé aux températures les plus basses pouvant donner un produit stérile et à des températures de 20 à $25^{\circ} \mathrm{F}$. plus élevées. Nous nous servions également de laits provenant d'autres sources et de différentes qualités. Avant la stérilisation, le lait subissait un préchauffage à $140^{\circ} \mathrm{F}$. et parfois à $180^{\circ} \mathrm{F}$. La méthode comprenait une stérilisation rapide en chauffant et refroisissant le produit dans 11 à 12 secondes et un embouteillage aseptique. Le lait stérile était emmagasiné à 40,70 et $100^{\circ}$ F. Nous l'analysions au point de vue $\mathrm{N}$ total, $\mathrm{N}$ non caséique, $\mathrm{N}$ caséique, $\mathrm{N}$ non protéique et ammoniacal. De plus, les analyses chimiques déterminaient la teneur en vitamine $\mathrm{A}$ et carotène, thiamine, acide ascorbique et phosphatase. L'épreuve à l'acide thiobarbiturique servait à étudier la stabilité des matières grasses. Cette épreuve repose sur une réaction comprenant la formation d'un complexe de couleur rouge quand le gras oxydé est acidifié et chauffé avec l'acide 2-thiobarbiturique. Nous pratiquions l'épreuve au peroxyde. Aussi, nous faisions des essais organoleptiques toutes les semaines au cours du premier mois qui suivait le traitement et ensuite tous les mois. Nous nous servions du système de pointage de l'American Dairy Science Association. Les échantillons étaient soumis en duplicata et parfois en triplicata à un jury de dégustateurs. Avant d'analyser les différents lots de lait, nous les vérifions au point de vue stérilité par un comptage sur plaques.

\section{Résultats}

Voici un bref résumé des expériences encore en cours. Bien que nous n'ayons pas encore fait d'études statistiques des résultats acquis, nous croyons pouvoir les interpréter tels quels avec assez de sûreté.

\section{Essais organoliptiques.}

Le lait stérilisé à près de $300^{\circ} \mathrm{F}$. avait un pointage de saveur 
de 39 immédiatement après le traitement. Au bout d'une semaine d'entreposage à $40^{\circ} \mathrm{F}$, il pointait 39,5 ; à $70^{\circ} \mathrm{F}, 37$; et à $100^{\circ} \mathrm{F}$., 36. Après 2, 3 et 4 semaines, les pointages se présentaient comme suit : $38,5-39$ à $40^{\circ} \mathrm{F}$, 37 à $70^{\circ} \mathrm{F}$, ; tandis cue le lait conservé à $100^{\circ} \mathrm{F}$. n'obtenait que $33-34$. Les points changeaient peu au cours des troisième, quatrième et cinquième mois, aux trois températures de conservation. En général, les saveurs passaient de légèrement de cuit à saveur de vieux, à oxydée. Les pointages des saveurs pour le lait stérilisé à $280^{\circ} 5 \mathrm{~F}$. affichaient les mêmes tendances. Les laits concentrés 3 -1 se comportaient à peu près de la même manière que les laits réguliers au point de vue de la saveur. Fraîchement reconstitué, le lait concentré conservé presque deux mois à $40^{\circ} \mathrm{F}$, pointait 39 ; et à trois mois, il obtenait 37-38. Conservé à $70^{\circ} \mathrm{F}$, il avait 38 à 38,5 points après une semaine, et $37-37,5$ après un mois; la saveur ne valait plus que $36-36,5$ après trois mois. Quant au produit entreposé à $100^{\circ} \mathrm{F}$., il lui fut accordé $37-37,5$ points après deux semaines et 34 à 35 après einq semaines.

Analyses des vitamines.

Le taux d'acide ascorbique décroit d'environ $15 \%$ dans le lait stérilisé à $300^{\circ} \mathrm{F}$. Après une semaine, la teneur avait baissé d'environ $40 \%$ à la température de conservation de $40^{\circ} \mathrm{F}$., de $60^{\circ}$ à $70^{\circ} \mathrm{F}$., et d'à peu près $80^{\circ} \%$ à $100^{\circ} \mathrm{F}$. Ces valeurs variaient peu au cours des troisième et quatrième semaines pour les trois températures. Après deux mois, le lait conservé à $40^{\circ} \mathrm{F}$. avait perdu environ $85 \%$ de son acide ascorbique et ceux gardés à 70 et à $100^{\circ} \mathrm{F}$., il ne leur en restait presque plus. La teneur en acide ascorbique du lait stérilisé à $280^{\circ} 5 \mathrm{~F}$. était pratiquement nulle après deux semaines d'entreposage. Le lait concentré $3-1$ perdait environ $65 \%$ de son acide ascorbique durant la stérilisation et n'en comptait presque plus après deux semaines aux trois températures de conservation.

La teneur en carotène et en vitamine A ne montre pas de changement significatif dans le lait chauffé à 280,5 ou à $300^{\circ} \mathrm{F}$., puis entreposé pendant trois mois à 40,70 et $100^{\circ} \mathrm{F}$. Les laits concentrés aux deux températures en ont perdu $10 \%$ durant la stérilisation sans autres pertes subséquentes au cours des trois mois aux trois températures.

La stérilisation a détruit $10 \%$ de la thiamine. Sa teneur a semblé augmenter pendant la conservation à 40 et à $70^{\circ} \mathrm{F}$. à cause probablement de substances interférentes ou fluorescentes formées au cours de cette période. La thiamine diminua pendant l'entreposage de trois mois à $100^{\circ} \mathrm{F}$. et sa teneur était presque toujours plus basse à cette température. La même tendance générale s'est manifestée avec le lait concentré. 
Jusqu'à maintenant, d'après les expériences poursuivies dans notre usine d'essai, les analyses indiquent que la stérilisation rapide du lait n'affecte pas sa valeur nutritive plus que ne le fait la pasteurisation à $143^{\circ} \mathrm{F}$, pendant 30 minutes, ou à $161^{\circ} \mathrm{F}$. pendant 15 secondes, et que la capacité de conservation de la saveur est grandement supérieure à celle du lait pasteurisé.

Analyses des proteines.

Les fractions d'azote non caséique, caséique et non protéique n'ont pas semblé avoir changé d'une façon significative durant les trois mois de conservation aux trois températures. La concentration en ions hydrogène des laits entreposés à $100^{\circ} \mathrm{F}$. durant trois mois a augmenté d'environ 0,25 unité $p \mathrm{H}$.

Des modifications se sont produites dans les fractions azotées du lait stérilisé à $280^{\circ} 5 \mathrm{~F}$. et conservé à 70 et $100^{\circ} \mathrm{F}$. L'azote caséique a décrû et l'azote non caséique a augmenté. Ce qui fut également vrai avec les laits concentrés $3-1$ entreposés à $100^{\circ} \mathrm{F}$. Cela nous fait croire à l'activité d'enzymes protéolytiques qui auraient survécu.

\section{Réactivation de la phosphatase.}

L'épreuve à la phosphatase du lait chauffé à $300^{\circ} \mathrm{F}$. et entreposé une semaine, donnait une lecture de $0,5 \gamma$ à $40^{\circ} \mathrm{F}$., $1,6 \gamma$ à $70^{\circ} \mathrm{F}$., et 1,3 à $100^{\circ} \mathrm{F}$. Ces valeurs ne variaient pas sensiblement au cours de la deuxième semaine de conservation mais durant la troisième le lait conservé à $70^{\circ} \mathrm{F}$. avait $5,2 \gamma$. Après quatre mois, les valeurs obtenues à la phosphatase étaient respectivement pour les trois températures de $2,9,14$ et 3,7 . Le lait conservé à $70^{\circ} \mathrm{F}$. a done donné des lectures plus élevées. La réactivation de la phosphatase se produisait plus vite dans le lait stérilisé à $280^{\circ} 5 \mathrm{~F}$. Après sept jours d'entreposage à $70^{\circ} \mathrm{F}$., le lait avait $12,8 \gamma$, et à $100^{\circ} \mathrm{F}$., 10,1 . Ces valeurs n'ont pas varié beaucoup durant trois mois. La réactivation de la phosphatase a été observée dans les laits concentrés après sept jours de conservation à $70^{\circ} \mathrm{F}$. et à $100^{\circ} \mathrm{F}$., donnant 10 et $12 \gamma$ respectivement. A $40^{\circ} \mathrm{F}$., elle n'était pas réactivée.

Ces résultats indiquent-ils une réactivation réelle de l'enzyme ou s'agit-il de réactions pseudo-phosphatasiques eausées par des composés interférents produits durant le court cycle de la stérilisation et durant la période d'entreposage ? L'évidence de la réactivation est circonstancielle à ce stage-ci des expériences. Mais nous avons trouvé que lorsque le lait stérilisé est de nouveau chauffé aux températures de pasteurisation, les réactions à la phosphatase deviennent négatives ou près de zéro. Nous espérons que les expériences en cours révéleront des informations décisives sur la réactivation de la phosphatase dans le lait stérilisé. 
Stabilité des matières grasses.

Les résultats à l'acide thiobarbiturique et au peroxyde ont toujours été les plus élevés dans les laits conservés à $100^{\circ} \mathrm{F}$., et les plus bas à $40^{\circ} \mathrm{F}$. Jusqu'à maintenant, nous ne sommes pas capables d'établir l'importance de ces analyses dans l'explication des modifieations de saveurs et autres changements chimiques produits durant l'entreposage. Les peroxydes, c'est connu, sont instables et ordinairement ne s'accumulent pas en grandes quantités mais se décomposent pour libérer l'oxygène, lequel peut catalyser d'autres réactions. Nous croyons que la détermination des peroxydes une fois la semaine n'est pas suffisante, particulièrement au cours des premiers jours de l'entreposage; plus fréquente, elle permettrait de recueillir plus d'informations utiles. Les résultats à l'acide thiobarbiturique ont correspondu étroitement avec l'apparition et le développement de la saveur d'oxydation dans le lait et la crème.

\section{Propriétés physiques.}

La viseosité du lait stérile augmente pendant la conservation à $40^{\circ} \mathrm{F}$. et à $70^{\circ} \mathrm{F}$. A $100^{\circ} \mathrm{F}$., le produit crème, formant des amas de gras qui nuisent à la détermination de la viscosité. Dans le lait conservé pendant plus de trois mois à $70^{\circ} \mathrm{F}$., il se produit une ennuyeuse montée du gras. Ce problème n'est pas sérieux pour le lait gardé à $40^{\circ} \mathrm{F}$. Après trente jours à $100^{\circ} \mathrm{F}$., la réaction du brunissement apparait dans le lait stérile et s'aggrave graduellement; cette réaction est plus intense dans le lait concentré.

Après une conservation de trente jours à $100^{\circ} \mathrm{F}$., le lait concentré prenait une structure de gel; après deux mois, celui conservé à $70^{\circ} \mathrm{F}$. affichait la même condition. Quant à celui gardé à $40^{\circ} \mathrm{F}$., il possédait un corps normal bien que sa viscosité ait augmenté. En industrie laitière, ce gel porte le nom d'épaississement de vieillesse. Quelques membres du jury ont noté le corps plus consistant du lait stérile, particulièrement après conservation à la plus basse température.

\section{Discussion des résultats}

Dans nos expériences, nous avons stérilisé du lait sans durée de retenue : ce qu'on pourrait appeler, bien que l'expression soit peu précise, chauffage instantané. Nous ignorons la vitesse d'échange de chaleur et le moment exact où, relativement à la température notée, les bactéries sporogènes sont détruites ou inactivées, mais nous pouvons dire que le lait, à un débit donné et à une température déterminée, est stérile. La construction d'un appareil à haute température et l'échange thermique qui s'y fait posent des problè- 
mes cruciaux au sujet desquels nous avons besoin de renseignements afin d'améliorer les méthodes de pasteurisation et de stérilisation des produits laitiers et autres denrées. Vous savez tous que les solides du lait ont une forte tendance à adhérer aux surfaces métalliques chauffées. Un progrès considérable s'est produit au cours des dernières années en vue de retarder cette action de cuisson en obligeant le lait à circuler à de grandes vitesses dans des tubes ou sur des surfaces de métal chauffées et en provoquant un écoulement tumultueux. Des recherches plus poussées sont nécessaires dans ce domaine. L'absorption des solides du lait sur les plaques des pasteurisateurs à hautes températures a toujours été un problème, particulièrement avec les laits de qualités inférieures chauffés à $170-180^{\circ} \mathrm{F}$.

Comme nous savons que le lait peut être pasteurisé en le chauffant à $161^{\circ} \mathrm{F}$., pendant 15 secondes, et puisque nous savons également pouvoir le stériliser à des températures inférieures à $300^{\circ} \mathrm{F}$., nous sommes done en face, pour la pasteurisation du lait et de quelques-uns de ses dérivés, d'une marge étendue des facteurs conjoints température-durée. Cette évidence paraît quand nous portons, en ordonnée d'un papier graphique, les logarithmes des durées (15.secondes et 30 minutes) de pasteurisation des standards officiels, et en asbcisse les températures ( $143^{\circ}$ et $161^{\circ} \mathrm{R}$.), supposent dans tous les cas un chauffage et un refroidissement instantanés. Avec un appareil capable d'atteindre rapidement de hautes températures, le lait et certains de ses dérivés peuvent subir la pasteurisation à des températures plus élevées que celles utilisées actuellement. Ils peuvent être pratiquement stérilisés entre 240 et $270^{\circ} \mathrm{F}$, toutes bactéries détruites, à l'exception des sporogènes les plus thermorésistantes; et le bon goût du lait pourra se conserver pendant un mois et probablement plus à $40^{\circ} \mathrm{F}$. Nos expériences sur le lait pasteurisé à des températures inférieures à celles de la stérilisation ont donné des résultats encourageants et les recherches se poursuivent.

Les résultats présentés ici indiquent la nature des problèmes soulevés dans la production des laits entier et concentré stériles. En général, ils sont surtout d'ordre physique et chimique et non biologique. En dépit de ces difficultés concernant le traitement, l'embouteillage et la mise en boîtes aseptiques, ces produits paraissent sur les marchés. Mais leur traitement et leur manutention paraissent difficiles et exigent un matériel compliqué, muni de contrôles précis pour la température et le débit, une attention toujours en éveil quant au lavage et à la stérilisation des appareils, et une destruction des bactéries de l'air. Mais nous prévoyons que ces opérations deviendront communes et seront contrôlées automa- 
tiquement, avec un système de chauffage plus efficace, muni d'instruments faibles et précis. Nous devons affirmer que des recherehes supplémentaires s'imposent sur les questions de base. Il en a été ainsi de pratiquement tous les produits laitiers et detoutes les denrées indépendamment de l'époque où ils furent fabriqués.

Les avantages des laits entiers et concentrés stériles peuvent se résumer ainsi :

$1^{0}$ Ils possèdent une meilleure qualité de conservation ;

$2^{\circ}$ Ils sont disponibles pour les pays et les régions où l'on a besoin de lait afin d'améliorer le niveau alimentaire des gens et où le lait pasteurisé régulier ne peut supporter les canaux de distribution ;

$3^{\circ}$ - Ils peuvent servir à l'approvisionnement des forces armées éloignées ;

$4^{0}$ Ils peuvent servir dans les distributeurs automatiques même si ces derniers sont installés loin de l'usine de traitement, chose impossible avec le lait simplement pasteurisé ;

$5^{0}$ Etant donné leur supériorité au point de vue conservation, ils peuvent suivre plus économiquement les voies de distribution que les produits non stériles;

$6^{\circ}$ Le lait peut être ramassé, stérilisé, embouteillé, dans une région laitière, puis distribué sans réfrigération par des moyens de transport moins dispendieux que ceux requis actuellement;

$7^{\circ}$ Le lait stérile est particulièrement avantageux dans l'alimentation des enfants à cause de sa commodité et parce qu'il conserve son bon goût longtemps;

$8^{\circ}$ Le lait peut être stérilisé et entreposé pendant les périodes de surproduction et utilisé lorsqu'il y a production insuffisante ;

$9^{\circ}$ Le lait stérile n'a pas à faire face aux problèmes bactériologiques que rencontre le lait simplement pasteurisé.

Pour conclure, nous pouvons dire que les progrès actuels en industrie laitière rendront plus facile et plus pratique la stérilisation du lait. Ces développements comprennent l'invention d'appareils améliorés de chauffage à haute température, les plus grandes facilités sur la ferme à produire du lait, l'apparition des bassins pour conserver, manipuler, et livrer en masse le lait à l'usine, les méthodes améliorées pour le nettoyage des appareils montés, les produits de lavage et de stérilisation de meilleure qualité, les recherches concernant l'effet de chaleur sur les propriétés et sur les constituants du lait, le travail des agents contrôleurs sur l'amélioration de la qualité des laits d'approvisionnement. Tous ces progrès contribuent grandement à hausser la qualité des laits crus, lesquels supporteront mieux les hautes températures de traitement de sorte que le produit 
fini se gardera plus longtemps qu'aujourd'hui. Il est généralement vrai que lorsqu'un procédé de traitement d'un aliment repose sur des bases de chimie, de physique et de mathématiques bien fondées et sur des principes de génie sains, qu'il sauvegarde les propriétés gustatives et nutritives du produit, il devient éventuellement économiquement possible et l'aliment qui en résulte prend sa place sur le marché au prix que le consommateur consent à payer. Nous possédons les appareils et les instruments nécessaires à la production de meilleurs produits laitiers actuels et nouveaux. Nous sommes à la veille de progrès plus considérables que jamais auparavant. De nouveaux horizons s'ouvrent pour l'industrie laitière.

\title{
REMERCIEMENTS
}

Ce travail a été possible grâce à un généreux octroi de l'Institut National de la Santé, aux collègues de l'Université de l'Illinois comprenant les professeurs Kaufmann, Tobias, Tracy, Tuckey, Whitney et Mile lalitha KadABA et M. V. L. SWEARINGEN qui ont aidé au cours des différentes étapes de cette étude. Les compagnies Anchor Hocking et Illinois Owen Glass ont fourni les contenants. Les compagnies Crown Cap et White Cap ont pourvu aux besoins de capsules et le chauffeur Mallory utilisé était celui de l'Tllinois Creamery Supply Co.

\section{PRÉPARATION DE PRÉSURE CRISTALLISÉE A PARTIR D'ESTOMACS DE VEAUX (CAILLETTES)}

\author{
par C. ALAIS
}

Station centrale de Microbiologie et Recherches laitières, Jouy-en-Josas.

Une méthode simple de préparation de présure cristallisée a été décrite par BERRIDGe et Woodward [1]. Ces auteurs ont employé comme matière première la présure commerciale de la maison anglaise Benger's Ltd. A la suite de l'échec de plusieurs tentatives de préparation, dans d'autres laboratoires à partir de produits différents, l'opinion a été émise que cette méthode ne pouvait réussir qu'avec la présure BENGER [2] et [3].

Nous avons été amenés à préparer la présure à l'état cristallisé afin de disposer d'une réserve d'enzyme pur, destiné aux recherches que nous poursuivons sur l'action de la présure sur la caséine. Dans la préparation décrite ei-dessous, nous sommes partis de caillettes du commerce, avec lesquelles nous avons obtenu une solution de présure, après un traitement simple et l'emploi de produits chimiques réduits au minimum pour avoir un liquide limpide et dont l'activité coagulante ou "force" soit à peu près celle de l'extrait

(1) Annales de Technologie (Ann. Inst. Nat. Rec. Agr., série E), 1955, nº 1, 113. 